\title{
Choke-free flow in trapezoidal channels with change in bed elevation
}

\author{
SUBHASISH DEY * and SOMNATH SIL \\ Department of Applied Mechanics, Regional Engineering College, \\ Durgapur 713209 , India \\ ${ }^{*}$ Present address: Department of Civil Engineering, Indian Institute of \\ Technology, Kharagpur 721302 , India \\ e-mail: sdey@civil.iitkgp.ernet.in \\ MS received 5 June 1997
}

\begin{abstract}
Solutions for choke-free flow in a trapezoidal channel, with rise in bed elevation that may occur with partial filling up of the channel bottom, are discussed. In this analysis, the side slopes of the channel are assumed to be the same before and after the transition. Considering smooth and gradual transition zones, equations of energy and continuity are solved for subcritical and supercritical upstream flow conditions to determine the maximum limiting rise in bed elevation. The ranges of the upstream flow depths are also obtained for the choke-free condition, using the continuity equation of the upstream flow.
\end{abstract}

Keywords. Open channel flow; choke-free flow; one-dimensional flow; steady flow; channel transition.

\section{Introduction}

The upstream flow of a trapezoidal channel is affected when the rise in bed elevation (figure 1) downstream of the transition zone exceeds a certain limit for a given approaching flow condition. This type of flow situation is said to be choked (Henderson 1966) for which no physical solution can be obtained mathematically. For designing a channel transition, it is essential to avoid the condition that causes choke. The phenomenon of choke in an open channel flow is completely dependent on the conditions of the flow before (upstream) and after (downstream) the transition influenced by the change in bed elevation. The critical flow in the downstream transition zone, where the Froude number of flow is unity, is the limiting condition of choke-free flow.

A list of symbols is given at the end of the paper 

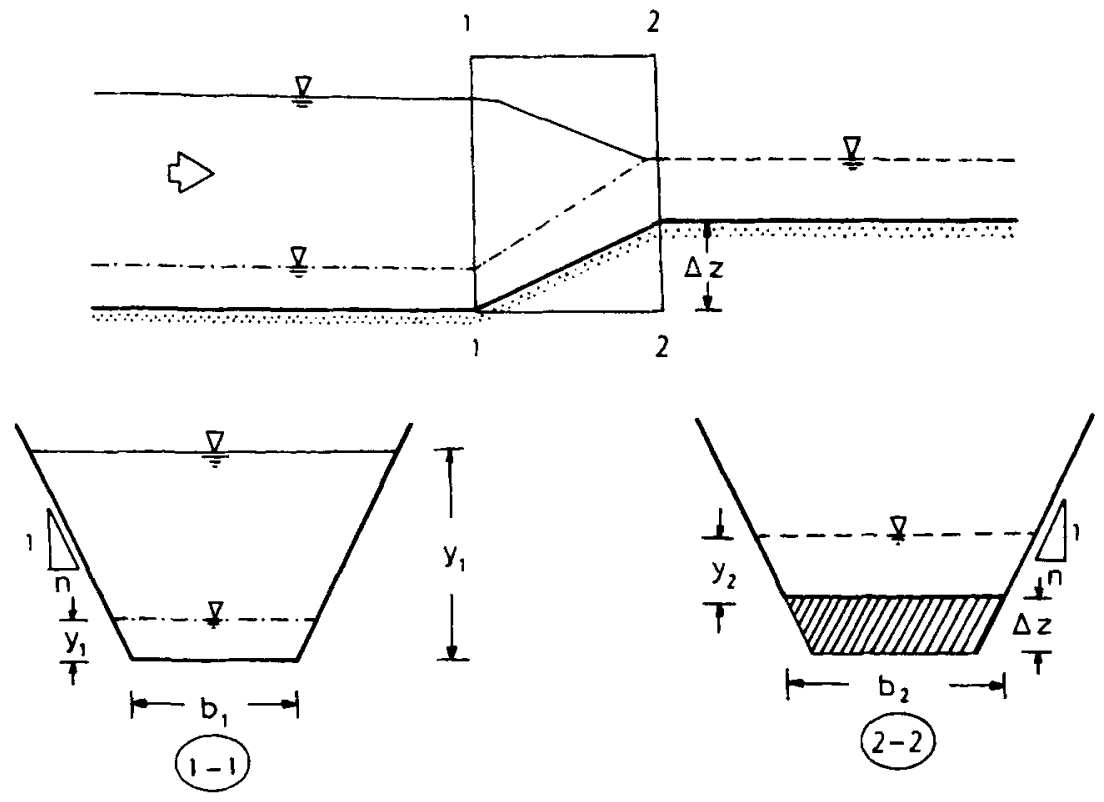

Figure 1. Definition sketch and hydraulic aspects. Flow profiles: - - critical, --- supercritical, - subcritical.

The solution of choke-free flow with a rise in bed elevation for a one-dimensional case was put forward by Allen (1980). Later Allen's method was applied by Liong (1984) in rectangular channels to obtain the solutions of choke-free flow due to changes in bed elevation and width. Dey et al (1990) gave generalized solutions of choke-free flow in triangular and parabolic channels for changes in bed elevation and shapes. Also, Dey (1994) presented the solutions for preventing choke in trapezoidal channels with a change in bed elevation considering that there was no change in channel sections upstream and downstream of the transition zone.

Considering a critical downstream flow, the equations of energy and continuity, upstream and downstream of the transition zone, are solved to determine the maximum permissible limits of change in bed elevation of the channel from the point of view of choke-free flow. Solutions of choke-free flow in trapezoidal channels are not straightforward and essentially involve the numerical solutions of implicit equations. Here, we consider an extension of the work of Dey (1994) to a different trapezoidal channel condition where the bed is raised owing to partial filling up of the channel bottom. The results are presented for the solutions of choke-free flow for such a case. In this analysis, the side slopes of the channel are considered to be the same before and after the transition zone which is assumed to be smooth and gradual, resulting in negligible loss.

\section{Governing equations}

The definition sketch for one-dimensional steady flow in a horizontally laid trapezoidal channel, whose bed is raised downstream of the transition zone, is shown in figure 1. Here, the side slopes of the channel are the same before and after the transition zone. The energy 
heads at sections 1 (before transition) and 2 (after transition) are equated, assuming that there is no loss of energy owing to the smooth and gradual transition zone,

$$
y_{1}+\alpha_{1}\left(V_{1}^{2} / 2 g\right)=y_{2}+\alpha_{2}\left(V_{2}^{2} / 2 g\right)+\Delta z .
$$

where $y=$ depth of flow, $\alpha=$ Coriolis coefficient assumed to be unity, $V=$ mean velocity of flow, $g=$ gravitational constant, and $\Delta z=$ bed elevation difference between sections 1 and 2 . Subscripts 1 and 2 indicate sections 1 and 2 respectively.

In an open channel flow, the Froude number of flow is given by $F=V / \sqrt{g D}$ (Chow 1959 ), where $D$ is the hydraulic mean depth. For trapezoidal channel, the hydraulic mean depth $D$ is given by flow area divided by the water surface width, or

$$
D=y(b+n y) /(b+2 n y)
$$

where $b=$ bottom width, and $n=$ side slope. The bottom widths in the upstream and downstream zones can be related as

$$
b_{2}=b_{1}+2 n \Delta z \text {. }
$$

Incorporating the Froude number into (1), the following quadratic equation in normalized form, which is used to determine the maximum allowable rise in bed elevation from the information on upstream flow parameters and channel geometry, is obtained:

$$
\begin{gathered}
\left(1+2 \hat{b} \hat{y}_{1}\right)\left(1-\hat{y}_{1}+0.5 F_{1}^{2} \frac{1+\hat{b}}{1+2 \hat{b}}\right) \hat{y}_{2}^{2}+\left[\left(2 \hat{b} \hat{y}_{1}\right)\left(1-\hat{y}_{1}+0.5 F_{1}^{2} \frac{1+\hat{b}}{1+2 \hat{b}}\right)\right. \\
\left.-\left(1+2 \hat{b} \hat{y}_{1}\right)\left(1+0.5 F_{2}^{2}\right) \hat{y}_{1}\right] \hat{y}_{2}-\left(2+0.5 F_{2}^{2}\right) \hat{b} \hat{y}_{1}^{2}=0,
\end{gathered}
$$

where $\hat{y}_{1}=\Delta z / y_{1} ; \hat{y}_{2}=\Delta z / y_{2} ;$ and $\hat{b}=\left(n y_{1}\right) / b_{1}$.

The continuity equation between sections 1 and 2 is

$$
V_{1}\left(b_{1}+n y_{1}\right) y_{1}=V_{2}\left(b_{2}+n y_{2}\right) y_{2} \text {. }
$$

Introducing the Froude number in (5) and normalizing it leads to

$$
\frac{F_{1}}{F_{2}}=\left[\frac{1+2 \hat{b}}{1+2 \hat{b}\left(1+\hat{y}_{2}\right)\left(\hat{y}_{1} / \hat{y}_{2}\right)}\right]^{0.5}\left[\frac{1+\hat{b}\left(1+2 \hat{y}_{2}\right)\left(\hat{y}_{1} / \hat{y}_{2}\right)}{1+\hat{b}}\right]^{1.5}\left(\frac{\hat{y}_{1}}{\hat{y}_{2}}\right)^{1.5} .
$$

$\hat{y}_{1}$ is solved from (4), ignoring the negative value, as

$$
\hat{y}_{1}=\left[-\xi_{2}+\left(\xi_{2}^{2}-4 \xi_{1} \xi_{3}\right)^{0.5}\right] /\left(2 \xi_{1}\right),
$$

where

$$
\begin{aligned}
& \xi_{1}=\left(1+2 \hat{b} \hat{y}_{1}\right)\left(1-\hat{y}_{1}+0.5 F_{1}^{2} \frac{1+\hat{b}}{1+2 \hat{b}}\right), \\
& \xi_{2}=\left(2 \hat{b} \hat{y}_{1}\right)\left(1-\hat{y}_{1}+0.5 F_{1}^{2} \frac{1+\hat{b}}{1+2 \hat{b}}\right)-\left(1+2 \hat{b} \hat{y}_{1}\right)\left(1+0.5 F_{2}^{2}\right) \hat{y}_{1}, \\
& \xi_{3}=-\left(2+0.5 F_{2}^{2}\right) \hat{b} \hat{y}_{1}^{2} .
\end{aligned}
$$


For choke-free flow, the limiting value of the downstream Froude number of flow is unity. Hence, for $F_{2}=1$, (7) is expressed as

$$
\tilde{y}_{1}=\left[-\tilde{\xi}_{2}+\left(\tilde{\xi}_{2}^{2}-4 \tilde{\xi}_{1} \tilde{\xi}_{3}\right)^{0.5}\right] /\left(2 \tilde{\xi}_{1}\right),
$$

where

$$
\begin{aligned}
& \tilde{y}=\hat{y} ; \quad \text { for } \quad F_{2}=1, \\
& \tilde{\xi}_{1}=\left(1+2 \hat{b} \tilde{y}_{1}\right)\left(1-\tilde{y}_{1}+0.5 F_{1}^{2} \frac{1+\hat{b}}{1+2 \hat{b}}\right), \\
& \tilde{\xi}_{2}=\left(2 \hat{b} \tilde{y}_{1}\right)\left(1-\tilde{y}_{1}+0.5 F_{1}^{2} \frac{1+\hat{b}}{1+2 \hat{b}}\right)-1.5 \tilde{y}_{1}\left(1+2 \hat{b} \tilde{y}_{1}\right), \\
& \tilde{\xi}_{3}=-2.5 \hat{b} \tilde{y}_{1}^{2} .
\end{aligned}
$$

The equation for choke-free flow in a trapezoidal channel with rise in bed elevation (figure 1), derived from (6), is

$$
F_{1}=\left[\frac{1+2 \hat{b}}{1+2 \hat{b}\left(1+\tilde{y}_{2}\right)\left(\tilde{y}_{1} / \tilde{y}_{2}\right)}\right]^{0.5}\left[\frac{1+\hat{b}\left(1+2 \tilde{y}_{2}\right)\left(\tilde{y}_{1} / \tilde{y}_{2}\right)}{1+\hat{b}}\right]^{1.5}\left(\frac{\tilde{y}_{1}}{\tilde{y}_{2}}\right)^{1.5} .
$$

The above equation is an implicit equation for $\tilde{y}_{1}$. Muller's method (Conte $\&$ de Boor 1987) can be used, as was done by Dey (1994), for solving the values of $\tilde{y}_{1}$. Hence, the maximum permissible limit of $\Delta z$ can be determined.

Again, to determine the allowable ranges of the upstream flow depth for an existing channel of the present type from the information on upstream channel geometry, rise in bed elevation and discharge, the Froude number is incorporated into (1) and (5) as before, and they are rearranged to obtain the following equations in normalized form:

$$
\begin{gathered}
(1+2 \hat{z})\left(1-\hat{y}_{1}+0.5 F_{1}^{2} \frac{\hat{y}_{1}+\hat{z}}{\hat{y}_{1}+2 \hat{z}}\right) \hat{y}_{2}^{2}+\left[(2 \hat{z})\left(1-\hat{y}_{1}+0.5 F_{1}^{2} \frac{\hat{y}_{1}+\hat{z}}{\hat{y}_{1}+2 \hat{z}}\right)\right. \\
\left.-(1+2 \hat{z})\left(1+0.5 F_{2}^{2}\right) \hat{y}_{1}\right] \hat{y}_{2}-\left(2+0.5 F_{2}^{2}\right) \hat{z} \hat{y}_{1}=0 \\
\frac{F_{1}}{F_{2}}=\left[\frac{\hat{y}_{1}+2 \hat{z}}{\hat{y}_{2}(1+2 \hat{z})+2 \hat{z}}\right]^{0.5}\left[\frac{\hat{y}_{2}(1+2 \hat{z})+\hat{z}}{\hat{y}_{1}+\hat{z}}\right]^{1.5}\left(\frac{\hat{y}_{1}}{\hat{y}_{2}}\right)^{2.5}
\end{gathered}
$$

where $\hat{z}=(n \Delta z) / b_{1}$.

Again, for the case of choke-free flow $\left(F_{2}=1\right), \hat{y}_{1}$ is solved from (12), ignoring the negative value, as

$$
\tilde{y}_{1}=\left[-\tilde{\zeta}_{2}+\left(\tilde{\zeta}_{2}^{2}-4 \tilde{\zeta}_{1} \tilde{\zeta}_{3}\right)^{0.5}\right] /\left(2 \tilde{\zeta}_{1}\right),
$$

where

$$
\tilde{\zeta}_{1}=(1+2 \tilde{z})\left(1-\tilde{y}_{1}+0.5 F_{1}^{2} \frac{\tilde{y}_{1}+\hat{z}}{\tilde{y}_{1}+2 \hat{z}}\right)
$$




$$
\begin{aligned}
& \tilde{\zeta}_{2}=(2 \hat{z})\left(1-\tilde{y}_{1}+0.5 F_{1}^{2} \frac{\tilde{y}_{1}+\hat{z}}{\tilde{y}_{1}+2 \hat{z}}\right)-1.5 \tilde{y}_{1}(1+2 \hat{z}), \\
& \tilde{\zeta}_{3}=2.5 \hat{z} \tilde{y}_{1} .
\end{aligned}
$$

Therefore, the equation for choke-free flow, for the present case, obtained from (13) is

$$
F_{1}=\left[\frac{\tilde{y}_{1}+2 \hat{z}}{\tilde{y}_{2}(1+2 \hat{z})+2 \hat{z}}\right]^{0.5}\left[\frac{\tilde{y}_{2}(1+2 \hat{z})+\hat{z}}{\tilde{y}_{1}+\hat{z}}\right]^{1.5}\left(\frac{\tilde{y}_{1}}{\tilde{y}_{2}}\right)^{2.5} \text {. }
$$

The equation of continuity at section 1 is

$$
Q=V_{1}\left(b_{1}+n y_{1}\right) y_{1},
$$

where $Q=$ discharge.

Incorporating the Froude number and normalizing (17) yields

$$
\hat{Q}=\left[\left(\tilde{y}_{1}+2 \hat{z}\right)^{0.5} \tilde{y}_{1}^{2.5}\right] /\left[\left(\tilde{y}_{1}+\hat{z}\right)^{1.5} F_{1}\right],
$$

where $\hat{Q}=\left(b_{1} \sqrt{g \Delta \bar{z}}\right) \Delta z / Q$.

Using (14), (16) and (18), the allowable ranges of $\tilde{y}_{1}$ for a given $\hat{Q}$ can be estimated by Muller's method (Conte \& de Boor 1987) as before.

\section{Results and discussion}

Solutions for the limiting condition of choke-free flow in a trapezoidal channel with a rise in bed elevation are obtained numerically, using Muller's method (Conte \& de Boor 1987), from the derived equations in the preceding section. The effect of the standing wave becomes pertinent when supercritical flow is deflected by the sides of the channel due to contraction (Henderson 1966). Here, this effect is not considered because rise in bed elevation does not produce a standing wave in supercritical upstream flow conditions.

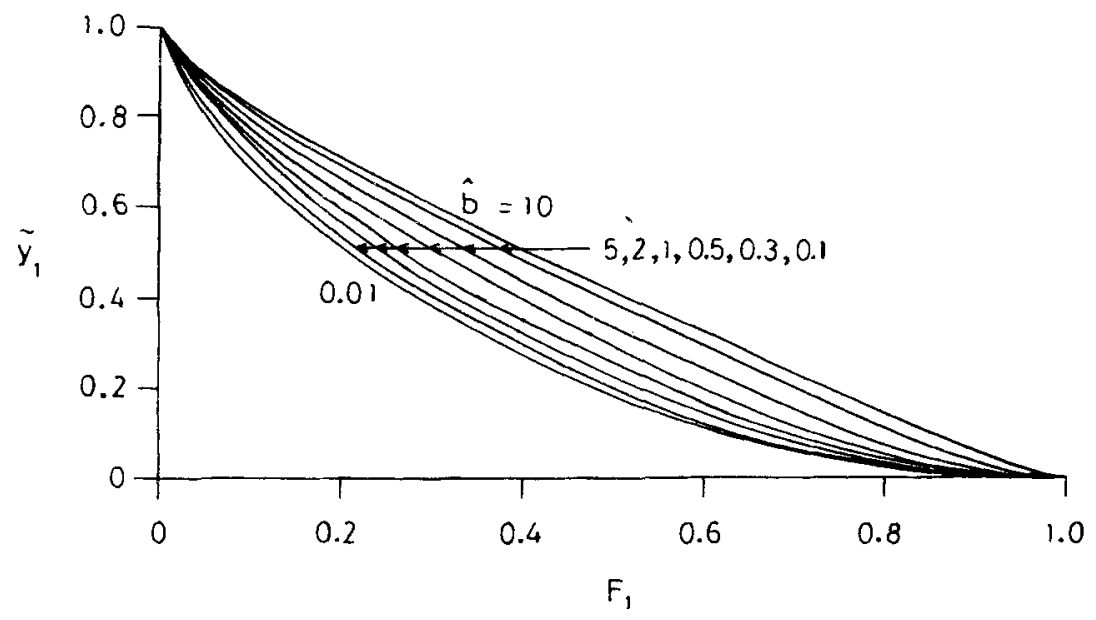

Figure 2. Curves for $\tilde{y}_{1}$ versus $F_{1}$ for different $\hat{b}$ in subcritical upstream flow conditions. 


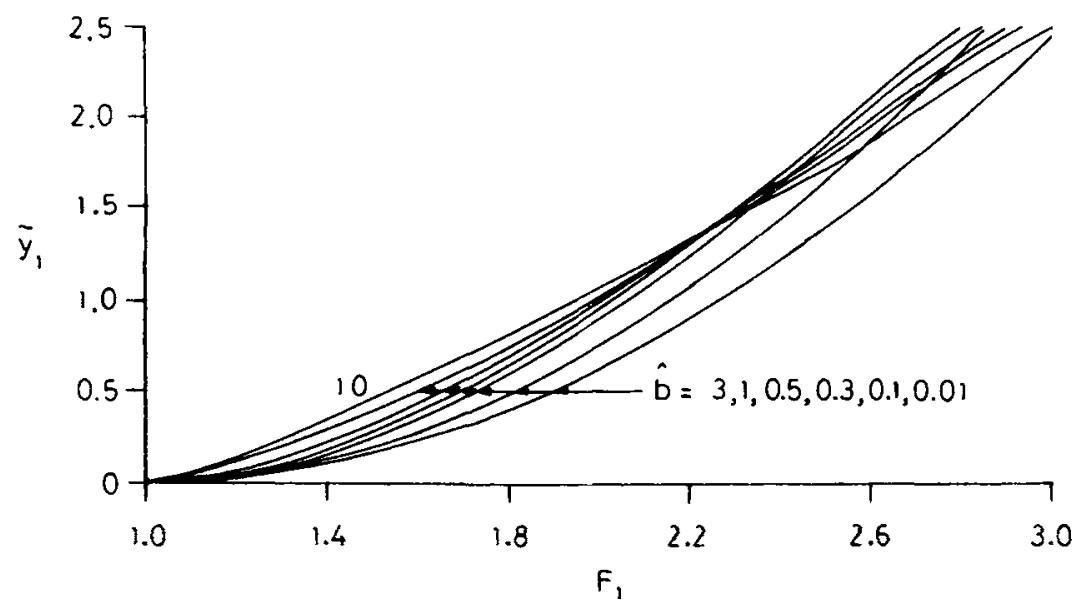

Figure 3. Curves for $\tilde{y}_{1}$ versus $F_{1}$ for different $\hat{b}$ in supercritical upstream flow conditions.

\subsection{Determination of $\Delta z$}

The variations of $\tilde{y}_{1}$ with $F_{1}$ for different $\hat{b}$ in subcritical and supercritical upstream flow conditions, obtained from the solution of (11), are shown in figures 2 and 3, respectively. In figure 3 , the upper limit of $F_{1}$ is restricted to three because $F_{1}>3$ is usually not a practical proposition in flow through an open channel. To determine the maximum limits of $\Delta z$ for avoiding choke flow, these curves can be used for design purposes. Hence, the designers should confine their chosen data to the choke-free regions which are the regions at the lower side of the curves in figures 2 and 3.

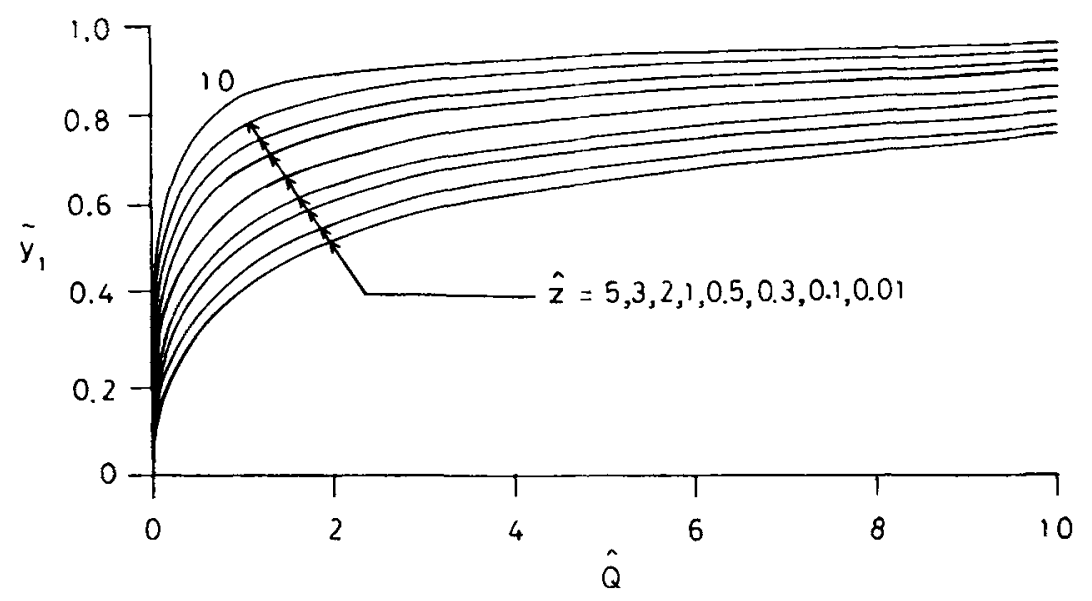

Figure 4. Curves for $\tilde{y}_{1}$ versus $\tilde{Q}$ for different $\hat{z}$ in subcritical upstream flow conditions. 


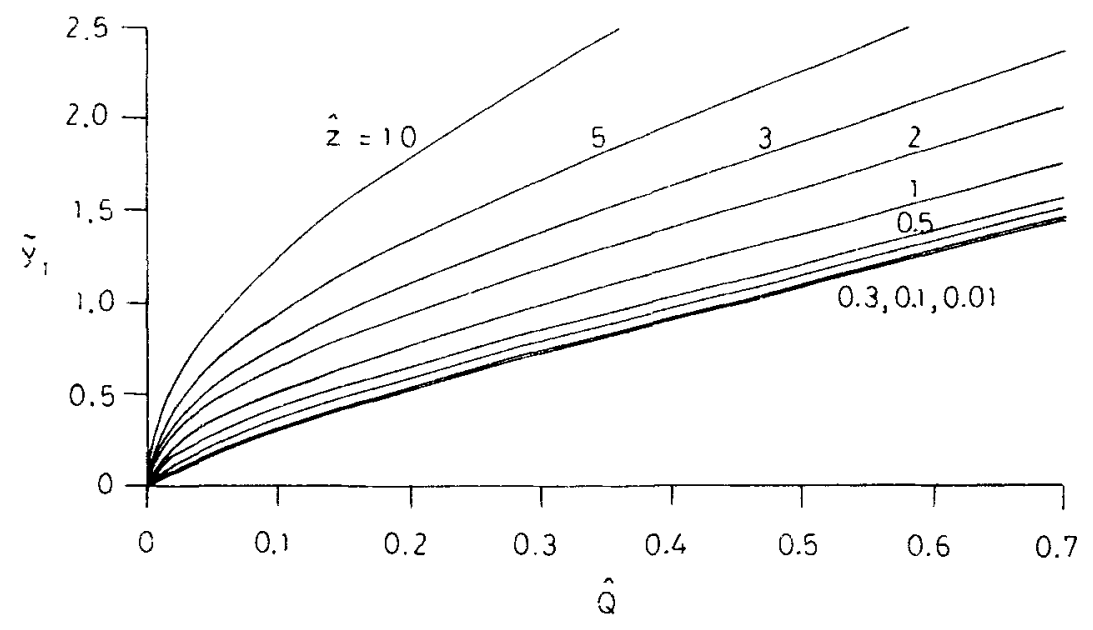

Figure 5. Curves for $\tilde{y}_{1}$ versus $\tilde{Q}$ for different $\hat{z}$ in supercritical upstream flow conditions.

\subsection{Determination of $y_{1}$}

The dependency of $\tilde{y}_{1}$ on $\hat{Q}$ for different $\hat{z}$ in subcritical and supercritical upstream flow conditions, obtained from the solution of (14), (16) and (18), are shown in figures 4 and 5 , respectively. The upper ranges of $\tilde{y}_{1}$ and $\hat{Q}$, in figure 5 , are restricted to 2.5 and 0.7 respectively, as the corresponding value of $F_{1}$ is greater than three. To determine the allowable ranges of $y_{1}$ to prevent choke, these curves can be utilized for the purpose of upstream flow operation. The choke-free regions, where the engineers should adjust the upstream flow depths, are the regions at the lower and upper sides of the curves in figures 4 and 5 respectively.

\section{Applications of the solution curves}

The present approach could be utilized, as a tool, to confirm the choke-free flow in a trapezoidal channel with rise in bed elevation. Hence, the proposed simple and convenient curves (figures 2-5) for choke-free flow are quite attractive. The use of the solution curves is illustrated by the following examples.

- The maximum permissible limit of $\Delta z$ is to be determined for choke-free conditions using the data $Q=3.068 \mathrm{~m}^{3} / \mathrm{s}, y_{1}=1 \mathrm{~m}, b_{1}=2 \mathrm{~m}$, and $n=2$.

Using the above data, one obtains $\hat{b}=1, V_{1}=0.767 \mathrm{~m} / \mathrm{s}$, and $F_{1}=0.30$, which refers to a subcritical upstream flow condition. Using figure 2 , the value of $\tilde{y}_{1}$ is found to be 0.51 . Hence, $\Delta z$ is to be selected as less than $0.51 \mathrm{~m}$ in order to prevent choke. Similarly the maximum limit of $\Delta z$ in case of a supercritical upstream flow condition can be obtained using figure 3 .

- The ranges of $y_{1}$ are to be determined for choke-free conditions using the data $Q=3.69$ $\mathrm{m}^{3} / \mathrm{s}, b_{1}=2 \mathrm{~m}, n=2$, and $\Delta z=0.50 \mathrm{~m}$. 
Using the given data, one gets $\hat{z}=0.50$, and $\hat{Q}=0.60$. From figures 4 and 5 , the values of $\tilde{y}_{1}$ are 0.48 and 1.39 for subcritical and supercritical upstream flow conditions respectively. Thus, to avoid choke the ranges of $y_{1}$ should be either greater than $1.042 \mathrm{~m}$ or less than $0.36 \mathrm{~m}$.

In the above, the data of the two problems are considered as is given in Dey (1994). It is shown that, in the present case, the results are significantly different from that obtained by Dey (1994).

\section{Concluding remarks}

One-dimensional steady flow in trapezoidal channels with rise in bed elevation has been analysed for choke-free conditions. Considering critical flow downstream of the transition zone, energy and continuity equations have been solved numerically to prepare the curves for determination of maximum permissible limits of rise in bed elevation (figures 2 and 3 ) and allowable ranges of upstream flow depths (figures 4 and 5). The curves can be utilized to design the change in bed elevation and to choose the operating ranges of upstream flow depths for choke-free flow in subcritical and supercritical upstream flow conditions.

The authors are grateful to Bimalundu Dey for his helpful suggestions during the preparation of this paper.

\section{List of symbols}

$b \quad$ Bottom width;

$\hat{b} \quad\left(n y_{1}\right) / b_{1}$;

$D$ hydraulic mean depth;

$F \quad$ Froude number of flow;

$g$ gravitational constant;

$n \quad$ side slope;

$Q$ discharge;

$\hat{Q} \quad\left(b_{1} \sqrt{g \Delta z}\right) \Delta z / Q$;

$V$ mean velocity of flow;

$y$ depth of flow;

$\tilde{y} \quad \hat{y}$ for $F_{2}=1$;

$\hat{y} \quad \Delta z / y$

$\hat{z} \quad(n \Delta z) / b_{1}$;

$\alpha \quad$ Coriolis coefficient;

$\Delta z$ rise in bed elevation.

\section{Subscripts}

1 upstream section;

2 downstream section. 


\section{References}

Allen R F 1980 Steady solution for river flow. J. Hydraul. Div., Am. Soc. Civ. Eng. 106: 608-611 Chow V T 1959 Open channel hydraulics (New York: McGraw-Hill)

Conte S D, de Boor C 1987 Elementary numerical analysis: An algorithmic approach (New York: McGraw-Hill)

Dey S 1994 No-choke flow in trapezoidal channels. J. Eng. Mech., Am. Soc. Civ. Eng. 120: 2224-2231

Dey S, Dey A, Bajpai S, Singh A K 1990 Design and flow operation of triangular and parabolic channels without choke. Indian J. Power River Valley Devel. 60: 71-74

Henderson F M 1966 Open channel flow (New York: McMillan)

Liong S Y 1984 Channel design and flow operation without choke. J. Irrig. Drain. Eng., Am. Soc. Civ. Eng. 110: 403-407 